\title{
CRITICAL SPEED MEASUREMENTS IN THE TEVATRON COLD COMPRESSORS
}

\author{
B. DeGraff, R. Bossert, A. Martinez, W. M. Soyars \\ Fermi National Accelerator Laboratory \\ Batavia, IL, 60510, USA
}

\begin{abstract}
The Fermilab Tevatron cryogenic system utilizes high-speed centrifugal cold compressors, manufactured by Ishikawajima-Harima Heavy Industries Co. Ltd. (IHI), for high energy operations. Nominal operating range for these compressors is 43,000 to 85,000 rpm. Past foil bearing failures prompted investigation to determine if critical speeds for operating compressors fall within operating range. Data acquisition hardware and software settings will be discussed for measuring liftoff, first critical and second critical speeds. Several tests provided comparisons between an optical displacement probe and accelerometer measurements. Vibration data and analysis of the 20 Tevatron ring cold compressors will be presented.
\end{abstract}

KEYWORDS: Cold Compressors, Data Acquisition System, Fermilab Refrigeration System, Vibrations

PACS: 07.20.Mc

\section{INTRODUCTION}

The Tevatron superconducting magnets are cooled by a hybrid cryogenic system that consists of a Central Helium Liquefier (CHL) and 24 satellite refrigerators. Each of the satellite refrigerators cools two $125 \mathrm{~m}$ magnet strings. The magnets are of a warm iron design. Lower operating temperatures allow the magnets to carry higher current without quenching, and consequently permit an increase in the energy of the particle beam in the accelerator.

A single stage cold compressor is used in each of the 24 satellite refrigerators to lower the operating temperature of the Tevatron accelerator. The primary machine is a high-speed centrifugal compressor supplied by IHI. A shroudless, cast aluminum impeller is driven by a $1.5 \mathrm{~kW}$ induction motor. The vertical rotor assembly consists of a steel shaft and squirrel cage type rotor. The assembly is supported radially by two identical selfacting foil gas bearings and axially by a spiral groove gas thrust bearing. 
Unique quench characteristics of individual magnets and variation in static heat load in each of the twenty four satellite refrigerators dictates different process pressure and flow rates of the cold compressors. Currently, the inlet pressure of the Tevatron cold compressors varies from 55 to $77 \mathrm{kPa}$, where steady state operating speed is in the range of 44,000 to 80,000 rpm [1].

System-wide cold compressor operations began in 2000. Since 2001, cold compressor operations have been required to support the Tevatron Run II physics program. Since January of 2000, there have been seven failures of the tape-style foil journal bearings. At the time of these failures, the bearings had not even reached half of their life-expectancy. The failure mode appears to be a breakdown of the gas cushion in the bearings resulting in the shaft rubbing on the brass bearing housings. These failures are very costly as many of the shafts were damaged beyond repair. Several Tevatron system explanations were postulated to explain these failures including liquid droplet intake due to high liquid level and contamination in the helium flow. In the failures during the last five years, none of these system explanations could be correlated with the compressor bearing failure.

Investigation into the natural vibration modes of the cold compressor shafts was considered as a possible explanation for these bearing failures. If the cold compressor was operated at or very close to its first or second natural vibration mode, significant additional energy would be deposited into the bearing which would account for the damage. The IHI documentation states that the first critical vibration mode would occur at $3000 \mathrm{rpm}$ and that the second critical vibration mode would occur at $10000 \mathrm{rpm}$. If these numbers, in particular the second critical vibration mode, were elevated from their original documentation into the operating range of the compressors, the early failure of the gas foil bearings could be explained.

A dynamic analysis and test program was previously conducted to learn more about the bearing operations. Testing on ambient air without the vacuum vessel showed a second critical speed of 40,000 rpm with a lift-off speed of 23,000 rpm [2]. In response to this information, cold compressor minimum speeds were elevated from 30,000 rpm to 43,000 rpm.

\section{DATA ACQUISITION}

Initial field investigation into the critical speeds of the Tevatron cold compressors utilized a portable oscilloscope and magnetically attached external accelerometer. Several attempts were made to sample a peak-hold vibration signature of startup, coast-down and steady-state operations.

Several curious observations appeared to be consistent across many hardware and software configurations. The amplitude of the vibration signature appears to increase linearly as the compressor's speed was increased from 40,000 rpm to 80,000 rpm. There also were repeatable spikes in the vibration amplitude at $815 \mathrm{~Hz}(48.9 \mathrm{krpm}), 1117 \mathrm{~Hz}$ $(67.0 \mathrm{krpm})$ and $1320 \mathrm{~Hz}(79.2 \mathrm{krpm})$. Instead of occurring at the natural frequencies of the compressor shaft, these values were located at the natural vibration frequencies of the casing hardware. This was discovered by analyzing the Fast Fourier Transforms (FFT) of a metal hammer striking the exterior of the compressor. The same frequency spikes were seen on the FFT with the compressor off.

This presented a significant problem when externally measuring the actual vibrations of the compressor shaft because these case natural frequencies were appearing as half and quarter natural frequencies whenever the compressor was operating in the lower speed range of 30 to $50 \mathrm{krpm}$. Any excitation of these harmonics created a level of background 
noise which completely dominated any real shaft signal present in the FFT or amplitude vs time graph.

The decision was made to modify one Tevatron cold compressor spare unit with two mini-accelerometers and one optical probe. The accelerometers would be located directly on the brass bearing housings and the optical probe would read the displacement of the thrust bearing plate edge. FIGURE 1 shows the approximate locations of the two accelerometers and the optical probe. The actual installation of the accelerometers is rotated $90^{\circ}$ away from the optical probe.

Three type 4507 Bruel \& Kjaer Miniature DeltaTron accelerometers were installed for comparing two internal vibration signatures with a third accelerometer attached to the external vacuum case. These accelerometers measured $10 \mathrm{~mm}$ x $10 \mathrm{~mm}$ x $16 \mathrm{~mm}$, which was just small enough to fit in the lower bearing housing external space. Grooves were machined in the brass housings and the accelerometers were epoxied in place. They have a sensitivity of $1 \mathrm{~V} / \mathrm{g}$ and a frequency range of 0 to $100000 \mathrm{~Hz}$. The optical probe is a Philtec model RC93-BEFv1T with a sensitivity of $7.11 \mathrm{E}-4 \mathrm{~mm} / \mathrm{mV}$. A grooved channel was machined in the motor housing and the probe was also epoxied in place. This instrumentation was installed on Tevatron spare cold compressor \#25.

For data acquisition, National Instruments (NI) software and hardware were purchased [3]. Portable signal conditioning modules were used to sample the accelerometer data. These modules have a $19000 \mathrm{~Hz}$ 3-pole Bessel lowpass hardware filter. A voltage input module was employed for the optical probe. These inputs were then fed into a laptop computer with a compatible NI-DAQ (Data Acquisition) PCMCIA card.

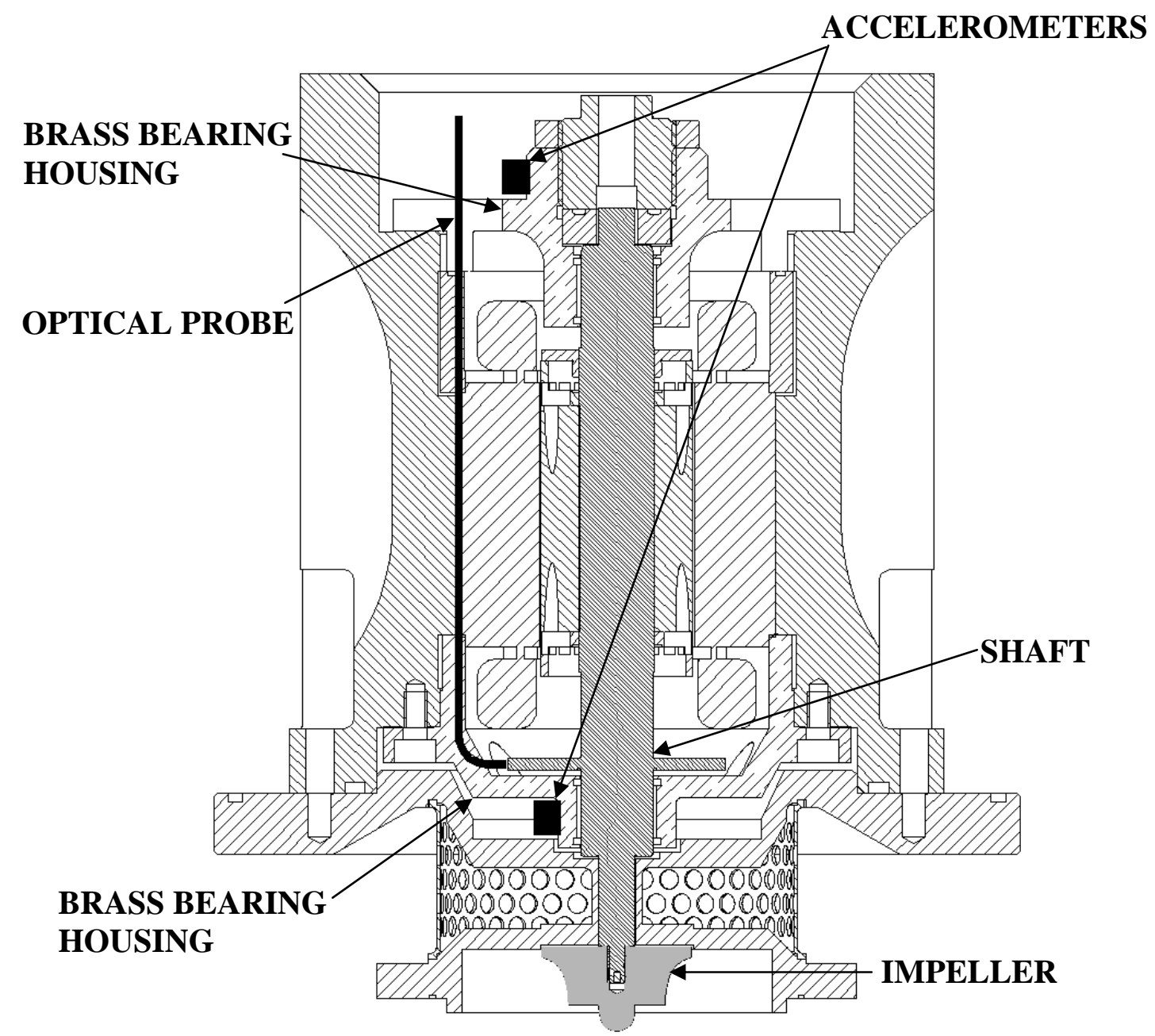

FIGURE 1. Cold Compressor Section View with Labels. 
NI Labview software was used to analyze the data. An additional piece of Labview software was purchased to assist in the analysis: Labview Advanced Signal Processing Toolset. This package of additional calculating tools allows for the use of the Joint Time Frequency Analysis.

\section{JOINT TIME FREQUENCY SIGNAL ANALYSIS}

Joint Time Frequency Analysis (JTFA) is a mathematical approach for expressing a signals time, frequency and amplitude simultaneously [4]. The desired results from JTFA on the cold compressors startups are the frequency at which the amplitude spikes during the 10 second startup from 0 to $43000 \mathrm{rpm}$. These frequencies would be the critical operating speeds of the compressors. Since the cold compressors are unable to operate for extended periods of time below $43000 \mathrm{rpm}$ due to bearing failure concerns, the only opportunity to view the vibration signature and record the critical speeds are during startups and coastdowns. A data acquisition difficulty arises given that these transient processes only last for 10 seconds. The sampling rate must be high enough to allow the JTFA around each time index.

One limitation of the Gabor Transform and other JTFA approaches is that the signal's time and frequency behaviors are not independent. When a signal's time duration gets narrower, its frequency bandwidth must become wider. The time duration and the frequency bandwidth cannot be made arbitrarily small simultaneously. This assertion is traditionally named the uncertainty principle [4]. The analysis inside Labview had to be tuned to produce the clearest results given this limitation.

Inside the Labview software, successful results were obtained by setting the time interval to 2048, the number of frequency bins to 2048 and limiting the frequency to 2000 Hz. The time interval is the number of samples to observe the input signal in the joint time frequency domain. The number of frequency bins specifies the exact number of frequency bins in the output time frequency representation.

\section{TEVATRON COLD COMPRESSOR RESULTS}

The first attempt at analyzing cold compressor \#25 vibration signature with the combined accelerometer, optical probe, NI hardware and Labview software was a partial success. The first few tests proved the concept that vibration and displacement readings could be obtained from inside the cold compressor. The major problem with these readings was the amount of extraneous transient signals that appeared in the JTFA output. This problem resulted from choosing a DAQ sampling frequency of $4000 \mathrm{~Hz}$. The original thinking was that the highest operating speed of the cold compressor corresponded to only $1333 \mathrm{~Hz}$ therefore sampling at 4000 would be above the Nyquist lower sampling limit [5].

The problem was discovered to be aliasing within the digital to analog converter of the NI DAQ card. Aliasing in a signal is defined as: "the erroneous interpretation of highfrequency signals as lower-frequency signals. Such misinterpretations are an expected result of making discrete measurements with sampling devices such as analog to digital converters [6].” These aliased signals present themselves as bouncing harmonics off the high and low frequency limit within the Gabor transform calculations.

To minimize the effects of aliasing on JTFA, the sampling frequency must be at least twice the hardware low-pass filter limit. Adding a low-pass filter inside Labview would not be sufficient since the digital to analog converter occurs in the NI hardware and not 
within the Labview software. The NI hardware was equipped to handle this aliasing problem since the input modules already possessed a $19 \mathrm{kHz}$ low-pass filter. Changing the sampling rate from $4000 \mathrm{~Hz}$ to $40000 \mathrm{~Hz}$ eliminated most of the contamination from the JTFA results.

The optical probe signal was analyzed with a $40000 \mathrm{~Hz}$ data acquisition rate inside cold compressor \#25. FIGURE 2 show the JTFA results from this testing. It indicates a liftoff speed of $15000 \mathrm{rpm}$, a first critical speed of $22000 \mathrm{rpm}$, and a second critical speed of $34000 \mathrm{rpm}$. Results from the upper and lower bearing accelerometers demonstrated similar results. The liftoff speed is defined as the speed at which the shaft displaces off the vertical thrust bearing. A change in the vibration signature of the compressor should be seen when the compressor's shaft begins floating on the gas film above the thrust bearing as the shaft is inherently less stable.

To obtain information for the operating Tevatron cold compressor, mounting clips were epoxied to the external vacuum vessel of all 20 compressors. The identical hardware used on compressor \#25 was then moved around the Tevatron ring to examine each compressor startup during the November 2004 Tevatron shutdown. FIGURE 3 shows the JTFA result from the cold compressor at the A2 house. The first critical speed was not identifiable but the liftoff and second critical could be identified. TABLE 1 shows the summary of results from the Tevatron ring. Houses B2 and C2 could not be analyzed, possibly due to poor signal transfer through the epoxy to the mounting clips.



FIGURE 2. \#25 Cold Compressor Optical Probe JTFA of 40 krpm Startup. 




FIGỦRE 3. Tevatron A2 Cold Compressor Accelerometer JTFA of 40 krpm Startup.

TABLE 1. Summary of Tevatron Cold Compress Critical Speed Survey.

\begin{tabular}{|c|c|c|c|c|}
\hline $\begin{array}{c}\text { TeV } \\
\text { FRIG } \\
\text { HOUSE }\end{array}$ & $\begin{array}{c}\text { IHI } \\
\text { MACHINE } \\
\text { NUMBER }\end{array}$ & $\begin{array}{c}\text { COMP } \\
\text { HOURS }\end{array}$ & $\begin{array}{c}\text { LIFTOFF } \\
\text { SPEED }\end{array}$ & $\begin{array}{c}\text { SECOND } \\
\text { CRITICAL } \\
\text { SPEED }\end{array}$ \\
\hline A1 & {$[$ \#] } & {$[\mathrm{hr}]$} & {$[\mathrm{rpm}]$} & {$[\mathrm{rpm}]$} \\
\hline A2 & 27 & 25000 & 15000 & 34000 \\
\hline A3 & 23 & 21000 & 15000 & 31000 \\
\hline B2 & 9 & 33000 & \multicolumn{2}{|c|}{ BAD DATA } \\
\hline B3 & 17 & 30000 & 14000 & 33000 \\
\hline B4 & 19 & 25000 & 14000 & 36000 \\
\hline C1 & 26 & 30000 & 15000 & 35000 \\
\hline C2 & 22 & 22000 & \multicolumn{2}{|c|}{ BAD DATA } \\
\hline C3 & 18 & 23000 & 10000 & 34000 \\
\hline D2 & 11 & 26000 & 14000 & 32000 \\
\hline D3 & 4 & 23000 & 15000 & 35000 \\
\hline D4 & 13 & 32000 & 9000 & 37000 \\
\hline E1 & 15 & 28000 & 15000 & 34000 \\
\hline E2 & 7 & 31000 & 15000 & 35000 \\
\hline E3 & 3 & 31000 & 15000 & 35000 \\
\hline E4 & 16 & 32000 & 15000 & 36000 \\
\hline F1 & 1 & 31000 & 14000 & 34000 \\
\hline F2 & 21 & 23000 & 15000 & 35000 \\
\hline F3 & 12 & 22000 & 15000 & 38000 \\
\hline F4 & 8 & 32000 & 14000 & 31000 \\
\hline CTF & 25 & 13000 & 15000 & 34000 \\
\hline & & & \multicolumn{3}{|c|}{} \\
\hline
\end{tabular}




\section{CONCLUSIONS}

Second critical speeds on installed IHI cold compressors have been measured, ranging from 31000 to $38000 \mathrm{rpm}$. Impeller liftoff speeds were typically 14000 to $15000 \mathrm{rpm}$. The presence of second critical speeds on the edge of the previous operating range, which had a lower limit of $30000 \mathrm{rpm}$, suggests that vibration played a role in the destruction of the gas foil bearings.

\section{ACKNOWLEDGEMENTS}

The authors are grateful to all those in the Cryogenic Department who have contributed to the testing and analysis of the Cold Compressors. Specifically Terry Cross and Bill Martin are thanked for their assistance in multiple days of testing on the cold compressors. Participants in the 2004 Turbomachinery Conference are thanked for their contributions in solving some DAQ problems relating to aliasing. Fermilab is operated by Universities Research Association Inc. under Contract No. DE-AC02-76CH03000 with the United States Department of Energy.

\section{REFERENCES}

1. Theilacker, J. C., Cryogenics 34, pp. 107-110 (1994).

2. Chen, H. M., Howarth, R., Geren, B., Theilacker, J. C., and Soyars, W. M. “Application of Foil Bearings to Helium Turbocompressors," in Proceedings of the Turbomachinery Symposium 30, edited by J.

Burnett, Turbomachinery Laboratory at Texas A\&M University, College Station, Texas, 2001, pp. 103114.

3. National Instruments Software, Labview Users Manual, http://www.ni.com, 2005.

4. Qian, Shie and Chen, Dapang, Joint Time-Frequency Analysis Methods and Applications, Prentice Hall PTR, New Jersey, 1996, CH 1 \& 2.

5. http://www.maxim-ic.com/appnotes.cfm/appnote_number/928

6. http://www.campbellsci.com/glossary.html 